\title{
1 The mycobacterial mutasome: composition and recruitment in live cells
}

2 Sophia Gessner ${ }^{1,2 \S}$, Zela Martin ${ }^{1,2,3 \S}$, Michael A. Reiche1,2,4§, Joana A. Santos ${ }^{5}$, Neeraj Dhar ${ }^{3, *}$, Ryan

3 Dinkele ${ }^{1,2}$, Timothy De Wet ${ }^{1,2,6}$, Atondaho Ramudzuli1,2, Saber Anoosheh, ${ }^{1,2,+}$ Dirk M. Lang7, Jesse

4 Aaron4, Teng-Leong Chew4, Jennifer Herrmann,9, Rolf Müller8,9, John D. McKinney33, Roger

Woodgate $^{10}$, Valerie Mizrahi1,2,11, Meindert H. Lamers5, Digby F. Warner ${ }^{1,2,11 \#}$

1SAMRC/NHLS/UCT Molecular Mycobacteriology Research Unit, DSI/NRF Centre of Excellence for

Biomedical TB Research, Department of Pathology, University of Cape Town, South Africa; ${ }^{2}$ Institute

8 of Infectious Disease and Molecular Medicine, University of Cape Town, South Africa; ${ }^{3}$ Laboratory of

9 Microbiology and Microsystems, School of Life Sciences, Swiss Federal Institute of Technology in 10 Lausanne (EPFL), Lausanne, Switzerland; ${ }^{4}$ Advanced Imaging Center, Howard Hughes Medical

11 Institute, United States of America; 5Department of Cell and Chemical Biology, Leiden University

12 Medical Center, The Netherlands; ${ }^{6}$ Department of Integrative Biomedical Sciences, University of Cape

13 Town, South Africa; ${ }^{7 C}$ Confocal and Light Microscope Imaging Facility, Department of Human Biology,

14 University of Cape Town, South Africa; ${ }^{8} \mathrm{Helmholtz} \mathrm{Institute} \mathrm{for} \mathrm{Pharmaceutical} \mathrm{Research} \mathrm{Saarland}$

15 (HIPS), Helmholtz Centre for Infection Research, Germany; ${ }^{9}$ German Centre for Infection Research

16 (DZIF), Partner Site Hannover-Braunschweig, Germany; ${ }^{10}$ Laboratory of Genomic Integrity, National

17 Institute of Child Health and Human Development, National Institutes of Health, United States of

18 America; ${ }^{11}$ Wellcome Centre for Infectious Diseases Research in Africa, University of Cape Town,

19 South Africa.

20 §These authors contributed equally

21 *Present address: Vaccine and Infectious Disease Organization (VIDO), University of Saskatchewan, 22120 Veterinary Road, Saskatoon, SK, S7N 5E3, Canada

23 tPresent address: Department of Chemistry and Umeå Centre for Microbial Research, Umeå 24 University, 90187, Umeå, Sweden.

25 \#For correspondence: digby.warner@uct.ac.za 


\section{ABSTRACT}

27 A DNA damage-inducible mutagenic gene cassette has been implicated in the emergence of drug 28 resistance in Mycobacterium tuberculosis during anti-tuberculosis (TB) chemotherapy. However, the

29 molecular composition and operation of the encoded "mycobacterial mutasome" - minimally 30 comprising DnaE2 polymerase and ImuA' and ImuB accessory proteins - remain elusive. Following 31 exposure of mycobacteria to DNA damaging agents, we observe that DnaE2 and ImuB co-localize with 32 the DNA polymerase III $\beta$ subunit ( $\beta$ clamp) in distinct intracellular foci. Notably, genetic inactivation

33 of the mutasome in an imuBAAAAGG mutant containing a disrupted $\beta$ clamp-binding motif abolishes 34 ImuB- $\beta$ clamp focus formation, a phenotype recapitulated pharmacologically by treating bacilli with 35 griselimycin and in biochemical assays in which this $\beta$ clamp-binding antibiotic collapses pre-formed 36 ImuB- $\beta$ clamp complexes. These observations establish the essentiality of the ImuB- $\beta$ clamp

37 interaction for mutagenic DNA repair in mycobacteria, identifying the mutasome as target for 38 adjunctive therapeutics designed to protect anti-TB drugs against emerging resistance. 


\section{INTRODUCTION}

40 Mycobacterium tuberculosis, the causative agent of tuberculosis (TB), consistently ranks among the

41 leading infectious killers worldwide (WHO, 2021). The heavy burden imposed by TB on global public

42 health is exacerbated by the emergence and spread of drug-resistant (DR) M. tuberculosis strains,

43 with estimates indicating that DR-TB now accounts for approximately one-third of all deaths owing

44 to antimicrobial resistance (Hasan 2018). In the absence of a wholly protective vaccine, a continually

45 replenishing pipeline of novel chemotherapeutics is required (Evans and Mizrahi 2018) which, given

46 the realities of modern antibiotic development (Nielsen 2019), appears unsustainable. Therefore,

47 alternative approaches must be explored including the identification of effective multidrug

48 combinations (Cokol et al., 2017), the elucidation of "resistance-proof" compounds (Ling 2015), and

49 the identification of so-called "anti-evolution" drugs that might limit the development of drug

50 resistance (Smith and Romesberg, 2007; Ragheb et al., 2019; Merrikh and Kohli, 2020).

51 Whereas many bacterial pathogens accelerate their evolution by sampling the immediate

52 environment - for example, via fratricide, natural competence, or conjugation (von Wintersdorff et

53 al., 2016; Veening and Blokesch, 2017) - these mechanisms appear inaccessible to M. tuberculosis:

54 the bacillus does not possess plasmids (Gray and Derbyshire, 2018) and there appears to be no role

55 for horizontal gene transfer in the modern evolution of strains of the M. tuberculosis complex

56 (Galagan, 2014; Boritsch and Brosch, 2016). Instead, genetic variation in M. tuberculosis results

57 exclusively from chromosomal rearrangements and mutations, a feature reflecting its ecological

58 isolation (an obligate pathogen, M. tuberculosis has no known host outside humans) and the natural

59 bottlenecks that occur during transmission (Gagneux, 2018). A question which therefore arises is

60 whether a specific molecular mechanism(s) drives M. tuberculosis mutagenesis - perhaps under

61 stressful conditions - and, consequently, if the activity thereof might be inhibited pharmacologically.

62 Multiple studies have investigated mycobacterial DNA replication and repair function in TB

63 infection models (for recent reviews, Singh, 2017; Minias et al., 2018; Mittal et al., 2020). From these,

64 the C-family DNA polymerase, DnaE2, has emerged as major contributor to mutagenesis under

65 antibiotic treatment (Boshoff et al., 2003). A non-essential homolog of E. coli DNA Polymerase (Pol)

66 III $\alpha$ (Timinskas et al., 2014), DnaE2 does not operate alone: the so-called "accessory factors", imuA'

67 and $i m u B$, are critical for DnaE2-dependent mutagenesis (Warner et al., 2010) (Figure 1A). Both

68 proteins are of unknown function, however $i m u A^{\prime}$ and $i m u B$ are upregulated together with dnaE2

69 following exposure of mycobacteria to DNA damaging agents including mitomycin C (MMC) (Figure

70 1B). This observation prompted the proposal that these three proteins might represent a 
71 "mycobacterial mutasome" - named according to its functional analogy with the E. coli DNA Pol V

72 mutasome comprising $\mathrm{UmuD}_{2}{ }_{2} \mathrm{C}$-RecA-ATP (Jiang et al., 2009; Erdem et al., 2014).

73 Here, we apply live-cell fluorescence and time-lapse microscopy in characterizing a panel of

74 mycobacterial reporter strains expressing fluorescent translational fusions of each of the known

75 mutasome components. The results of these analyses, together with complementary in vitro

76 biochemical assays utilizing purified mycobacterial proteins, support the inference that ImuB serves

77 as a hub protein, interacting with the dnaN-encoded $\beta$ clamp and ImuA'. They also reinforce the

78 essentiality of the ImuB- $\beta$ clamp protein-protein interaction for mutasome function. Notably, while

79 a strong ImuA'-ImuB interaction is detected in vitro, we report live-cell data which indicate the

80 dispensability of either ImuA' or DnaE2 for ImuB localization - but not mutasome function - in bacilli

81 exposed to genotoxic stress. Finally, using the $\beta$ clamp-binding antibiotic, griselimycin (GRS) (Kling

82 et al., 2015), we demonstrate in biochemical assays and in live mycobacteria the capacity to inhibit

83 mutasome function through the pharmacological disruption of ImuB- $\beta$ focus formation. These

84 observations suggest that, through its inhibition of $\beta$ clamp binding, GRS naturally limits the capacity

85 for induced mutagenesis. Therefore, as well as revealing a built-in mechanism protecting against

86 auto-induced mutations to GRS resistance, our results support the potential utility of "anti-evolution"

87 antibiotics for TB.

\section{RESULTS}

89 ImuB forms distinct sub-cellular foci under DNA damaging conditions.

90 Our previous genetic evidence (Warner et al., 2010) informed a tentative model in which the

91 catalytically inactive $Y$ family Pol homolog, ImuB, functioned as an adapter protein. According to the

92 model, DnaE2 gains access to the repair site by interacting with ImuB, which similarly interacts with

93 ImuA' and the dnaN-encoded $\beta$ clamp subunit (Figure 1A). To investigate the subcellular

94 localizations of each of the mutasome proteins in live bacilli, we constructed reporter alleles in which

95 the M. smegmatis mutasome proteins were labelled by N-terminal translational attachment of either

96 Enhanced Green (EGFP) or Venus Fluorescent Protein (VFP) tags (Figure 1 - figure supplement 1A).

97 The reporter alleles were introduced into each of three individual M. smegmatis mutasome gene

98 deletion mutants - $\triangle d n a E 2, \Delta i m u A^{\prime}$, and $\triangle i m u B$ (Warner et al., 2010) - to yield the complemented

99 strains, $\triangle d n a E 2$ attB::egfp-dnaE2 (designated G-dnaE2), $\triangle i m u B$ attB::egfp-imuB (G-imuB), and

$100 \Delta i m u A^{\prime}$ attB::vfp-imuA' (V-imuA'). 
bioRxiv preprint doi: https://doi.org/10.1101/2021.11.16.468908; this version posted November 17, 2021. The copyright holder for this preprint (which was not certified by peer review) is the author/funder, who has granted bioRxiv a license to display the preprint in perpetuity. It is made available under aCC-BY-ND 4.0 International license.

A

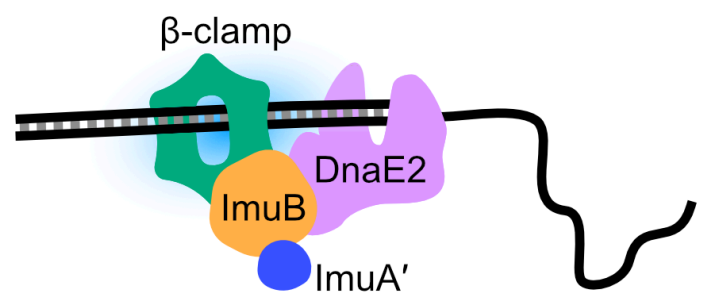

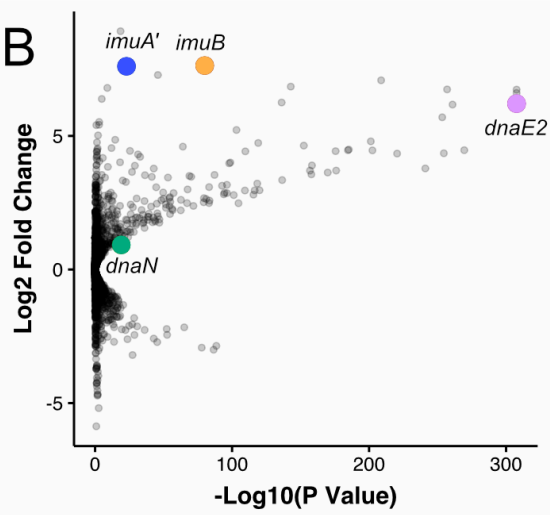

Overlay
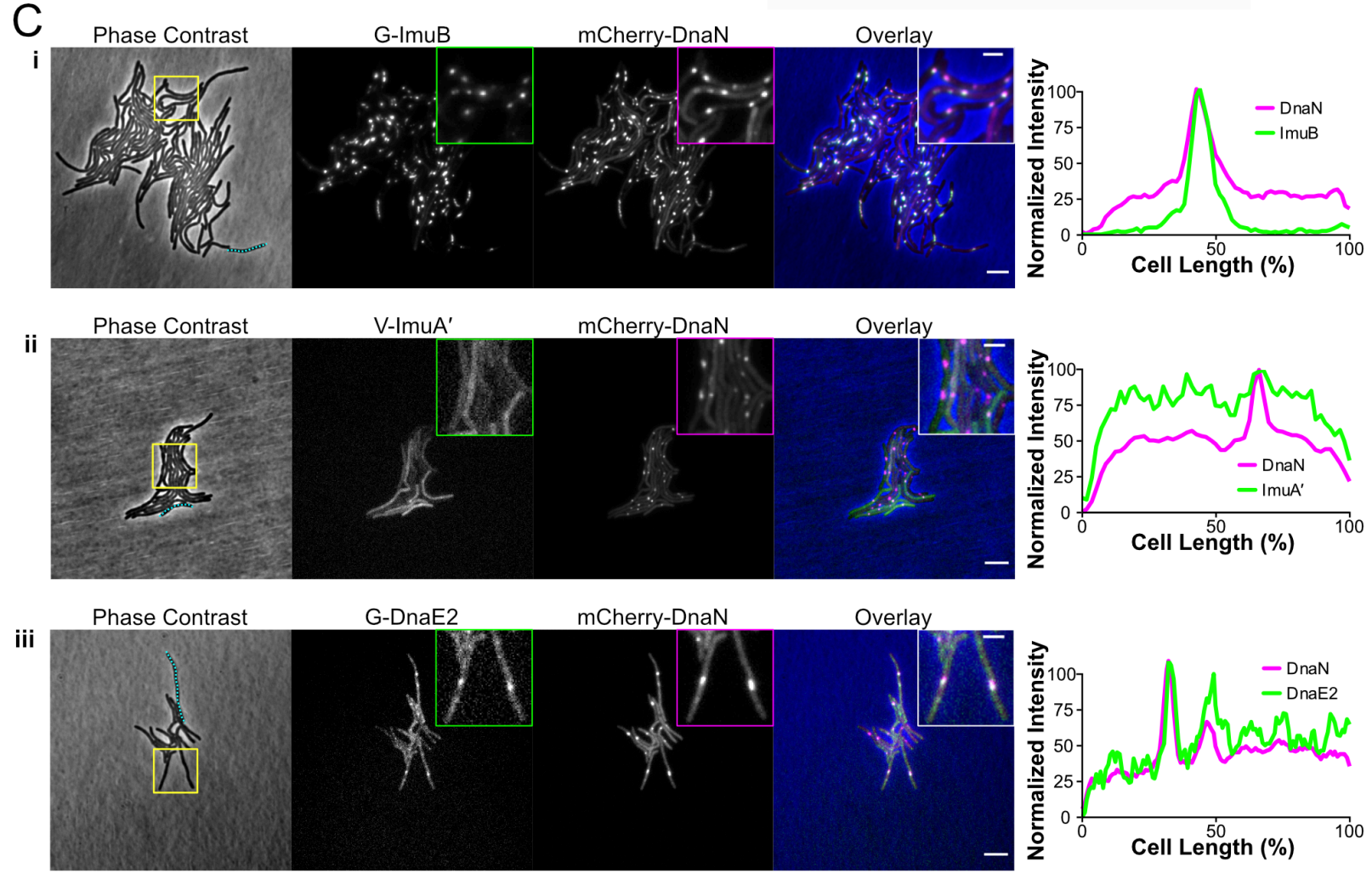

Figure 1. Components of the mycobacterial mutasome. A. Cartoon summarizing the available genetic, microbiological and bioinformatic data (Boshoff et al., 2003; Warner et al., 2010; Timinskas and Venclovas, 2019) into a working model of predicted mutasome composition. This model proposes that ImuB (blue) functions as an adapter protein, binding the dnaN-encoded $\beta$ clamp (orange) to enable access of the errorprone DnaE2 subunit (magenta) to the DNA replication fork. ImuB has also been predicted to bind accessory protein ImuA' (yellow). B. A volcano plot depicting transcriptional data from Müller et al. (2018) which were derived from RNA-seq analyses of wild-type $M$. smegmatis $m^{2} 155$ exposed to $1 \times M I C$ Mitomycin C (MMC). Consistent with corresponding observations in M. tuberculosis (Boshoff et al., 2003; Warner et al., 2010), the mutasome components, imuA', imuB, and dnaE2 are among the 15 most highly upregulated mycobacterial genes following $M M C$ exposure. C. Representative stills from single-cell timelapse fluorescence microscopy experiments of $M$. smegmatis expressing translational reporters of the different mutasome components. Phase contrast and fluorescence images of $M$. smegmatis expressing $G$ ImuB and mCherry-DnaN, V-ImuA' and mCherry-DnaN, and G-DnaE2 and mCherry-DnaN are represented following $4 \mathrm{~h}$ exposure to $1 / 2 \times M I C$ MMC. Scale bars in the overlay image represent $5 \mu \mathrm{m}$. Inset images at the top right corner of the fluorescence and overlay images show a zoomed-in region corresponding to the yellow box in each phase contrast image (inset scale bar represents $2 \mu \mathrm{m}$ ). The right-hand panel illustrates 
normalised fluorescence intensity along the longitudinal axis (as percentage [\%] of the total cell length) of a representative cell from each strain; the cell analysed is indicated by the blue dotted lines in the corresponding phase contrast image. Single-cell time-lapse fluorescence microscopy experiments were repeated 2-4 times; a typical experiment collected images from approximately 80 XY points.

The mycobacterial DNA damage response was induced by exposing the strains to the natural product antibiotic, mitomycin C (MMC), an alkylating agent that causes monofunctional DNA adducts and inter-strand and intra-strand cross-links (Bargonetti et al., 2010). Following exposure of V-imu $A^{\prime}$ to MMC for 4 hours, a yellow fluorescence signal was observable throughout the cells (Figure 1C, Figure 1 - figure supplement $1 B$ ), suggesting diffuse distribution of the VFP-ImuA' protein in the mycobacterial cytoplasm. In contrast, distinct EGFP-ImuB foci were observed in G-imuB bacilli following MMC treatment (Figure 1C, Figure 1 - figure supplement 1B). Although less distinct, EGFP-DnaE2 produced similar evidence of focus formation in G-dnaE2 cells (Figure 1C, Figure 1 figure supplement $1 B$ ). Notably, the significant increase in signal detectable in $\mathrm{V}$-imu $A^{\prime}, \mathrm{G}-\mathrm{imu} B$, and G-dnaE2 for MMC-exposed versus unexposed cells (Figure 1 - figure supplement 1B) confirmed that expression of the respective fluorescence reporter alleles was DNA damage-dependent in all three reporter mutants.

To determine whether these observations were also true for other types of DNA damage, the three reporter mutants were subjected to ultra-violet (UV) light exposure. Equivalent fluorescence phenotypes were observed for each of the three reporter alleles under both DNA damaging treatments (Figure 1 - figure supplement 1B). As UV exposure causes cyclobutane pyrimidine dimers or pyrimidine-pyrimidone (6-4) photoproducts (Boshoff et al., 2003), while MMC generates inter-strand DNA cross-links at CpG sites (Tomasz, 1995), these results indicated that expression and localization (recruitment) of the mutasome components might be independent of the nature of the genotoxic stress applied.

\section{$\mathrm{N}$-terminal fluorescent reporters retain wild-type mutagenic function but are deficient in DNA} damage tolerance.

The addition of bulky fluorescent tags can disrupt the function of DNA replication and repair proteins (Renzette et al., 2005). To determine whether any of the tagged mutasome proteins were affected, the functionalities of the egfp-imuB, $v f p$-imuA' and egfp-dnaE2 alleles were assessed in two standard assays (Boshoff et al., 2003; Warner et al., 2010): the first investigated DNA damage-induced mutagenesis following UV irradiation, and the second tested DNA damage tolerance following treatment with MMC. As observed previously (Boshoff et al., 2003; Warner et al., 2010), exposure of the wild-type parental M. smegmatis $\mathrm{mc}^{2} 155$ to a sub-lethal dose of UV irradiation increased the frequency of rifampicin (RIF) resistance 50 - to 100-fold, as determined from enumeration of colony 
forming units (CFU) on RIF-containing solid growth medium. In contrast, induced mutagenesis was greatly reduced in the $\Delta i m u A^{\prime}, \Delta i m u B$, and $\triangle d n a E 2$ deletion mutants: mutation frequencies for these "mutasome-deficient" strains were approximately 20-fold lower than wild-type (Figure 1 - figure supplement 2A). Complementation with the cognate fluorescent reporter allele in $\mathrm{V}$-imuA', G-imuB and G-dnaE2 restored the UV-induced mutation frequency of each of the three knockout mutants to near wild-type levels, establishing that each of the fluorescence reporter alleles retained function in UV-induced mutagenesis assays.

Surprisingly, the DNA damage tolerance assay - in which CFU forming ability is tested during continuous exposure to MMC in solid medium - produced contrasting results (Figure 1 - figure supplement $2 B$ ): unlike in the mutagenesis assay, complementation of either imu $A^{\prime}$ or imuB gene deletion mutants with their corresponding fluorescent reporter alleles failed to restore the wild-type phenotype, whereas dnaE2 hypersusceptibility was reversed. The reason for these discrepant observations - restoration of UV-induced mutagenesis but not MMC-induced DNA damage tolerance

- in the imuA and imuB strains is not clear. It seems likely that the different types of DNA damage induced by the two separate treatments might require distinct repair pathways and, potentially, discrete protein interactions which were differentially disrupted by the presence of a fluorescent tag on either mutasome component. Another possibility is that this phenotype was caused by the persistent/recurring damage sustained by the bacilli throughout the 4-day incubation on MMCcontaining medium - dissimilar to the comparatively short duration of UV exposure. However, these explanations are speculative and require further investigation.

\section{ImuB localizes with the $d n a N$-encoded $\beta$ clamp following DNA damage.}

We previously inferred that a putative interaction between ImuB and the $d n a N$-encoded $\beta$ clamp was essential for mutasome function (Warner et al., 2010). To investigate the predicted interaction of ImuB and the $\beta$ clamp in live bacilli, each of the three mutasome reporter alleles was introduced separately into an $M$. smegmatis mutant encoding an mCherry-tagged $\beta$ clamp, mCherry-DnaN (Santi et al., 2013). The mCherry-DnaN reporter was chosen as background strain owing to its previous validation in single-cell time-lapse fluorescence microscopy analyses of $M$. smegmatis replisome location (Santi et al., 2013; Santi and McKinney, 2015). For the time-lapse experiments, the $M$. smegmatis dual reporter strains were grown in standard 7H9/OADC medium for 12 hours, following

182 treatment recovery (Figure 1C; Figure 2; Videos 1-3). At 4 hours post MMC treatment, distinct EGFP183 ImuB foci were observed (Figure 1C panel i) which, when overlaid with the mCherry-DnaN 184 fluorescence signal, showed considerable overlap, suggesting association of the $\beta$ clamp with ImuB. 
185 Notably, the EGFP-ImuB signal was almost exclusively detected in very close proximity to mCherry-

186 DnaN foci, with very rare instances of EGFP signal detectable in regions where fluorescence was

187 absent. This association was independent of the duration of MMC exposure, occurring at all time

188 points tested (Figure 2A; Video 1). In combination, these results support the direct physical

189 interaction of ImuB and the $\beta$ clamp, as suggested previously by yeast two-hybrid and site-directed

190 mutagenesis studies (Warner et al., 2010).

191 For V-ImuA', a diffuse fluorescence signal was detected throughout the cells (Figure 1C panel

192 ii; Figure 2B; Video 2), rendering impossible any conclusion about the potential recruitment of ImuA'

193 to $\beta$ clamp (mCherry-DnaN) foci. In contrast, the results for DnaE2 were more nuanced: overlap of

194 peak fluorescence signals from EGFP-DnaE2 and mCherry-DnaN proteins was detected (Figure 1C

195 panel iii; Figure 2C) and was most evident within 1 hour post removal of MMC from the microfluidic

196 chamber (Video 3). Although not as consistent as the ImuB- $\beta$ clamp phenotype, the co-localization

197 was reproducibly observed in multiple cells and across different experiments.

198 ImuA' and DnaE2 are not required for ImuB focus formation.

199 We showed previously that deletion of $i m u A^{\prime}$ phenocopied abrogation of either imuB or

200 dnaE2 in the MMC sensitivity assay (Warner, et al., 2010) and, consistent with the interpretation that

201 all three components are individually essential for mutasome activity, this phenotype was not

202 exacerbated in a triple knockout mutant $\left(\triangle i m u A^{\prime}-i m u B \Delta d n a E 2\right)$ lacking all three mutasome

203 components. Moreover, yeast two-hybrid results predicted a direct interaction between ImuB and

204 ImuA' (Warner et al., 2010). Together, these observations suggested that a deficiency in ImuA' might

205 impair ImuB protein localization. To test this prediction, the egfp-imuB allele was introduced into the

$206 \Delta i m u A^{\prime}$ deletion mutant, generating a $\triangle i m u A^{\prime}$ attB::egfp-imuB reporter strain. Despite the loss of

207 ImuA' in this mutant, EGFP-ImuB foci were observed following MMC treatment (Figure 2 - figure

208 supplement 1i), mimicking the wild-type phenotype. The absence of functional DnaE2 similarly had

209 no discernible impact on ImuB focus formation in the corresponding catalytically dead dnaE2 AIA

210 attB::egfp-imuB or DnaE2-deleted $\triangle d n a E 2$ attB::egfp-imuB, mutants (Figure 2 - figure supplement

211 1ii \& iii). In combination, these results appear to eliminate a role for either ImuA' or DnaE2 in ImuB

212 localization, instead implying the critical importance of the ImuB- $\beta$ clamp interaction for mutasome

213 function. 
bioRxiv preprint doi: https://doi.org/10.1101/2021.11.16.468908; this version posted November 17, 2021. The copyright holder for this preprint (which was not certified by peer review) is the author/funder, who has granted bioRxiv a license to display the preprint in perpetuity. It is made available under aCC-BY-ND 4.0 International license.

A. G-ImuB (green), mCherry-DnaN (magenta)

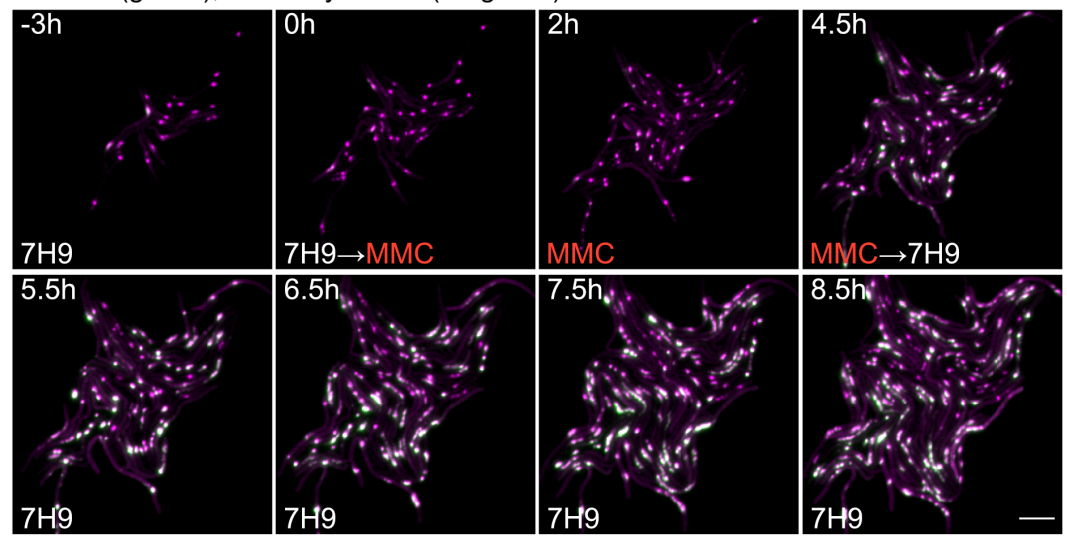

V-ImuA' (green), mCherry-DnaN (magenta)

\begin{tabular}{l|l|l} 
B. $-3 \mathrm{~h}$ & $\mathrm{Ch}$ & $2 \mathrm{~h}$
\end{tabular}

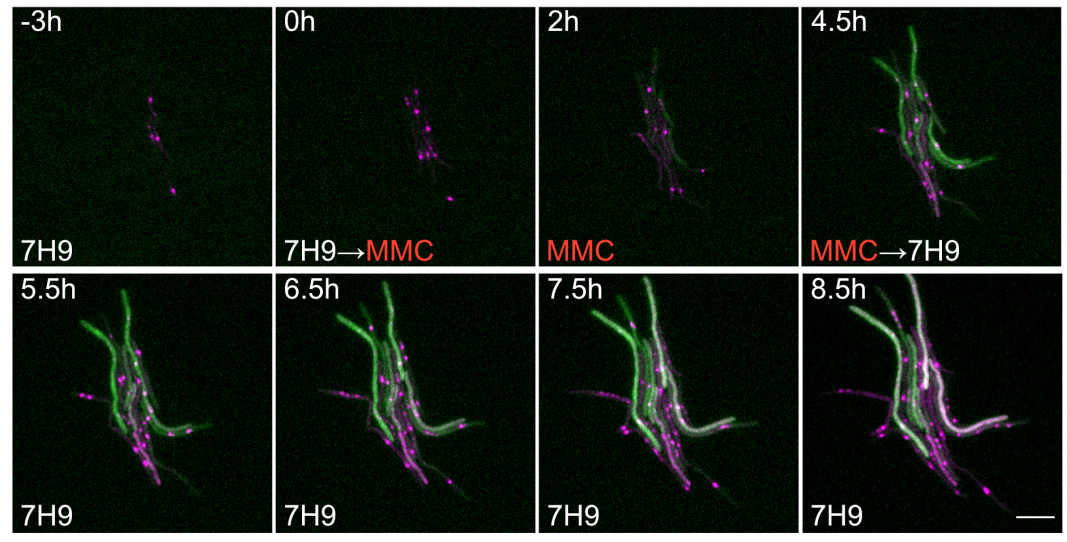

G-DnaE2 (green), mCherry-DnaN (magenta)

C.

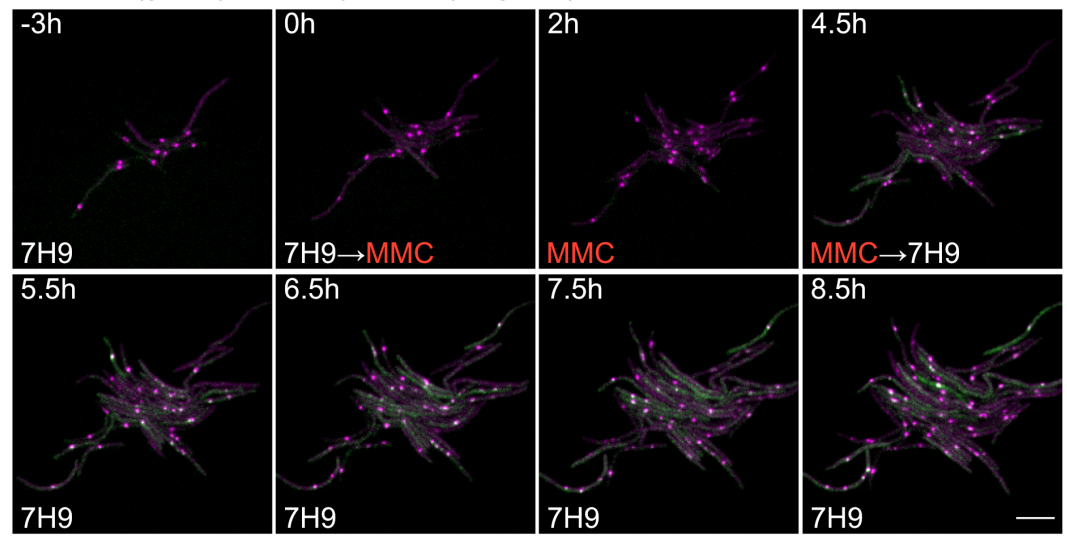

Figure 2. Representative time-lapse series of single-cells of M. smegmatis expressing the generated translational reporters in combination with mCherry-DnaN. A. G-ImuB (green) and mCherry-DnaN (magenta), B. V-ImuA' (green) and mCherry-DnaN (magenta), and C. G-DnaE2 (green) and mCherry-DnaN (magenta). Overlapping signals are viewed as white. The cells were exposed to MMC $(1 / 2 \times M I C)$, from time $0 \mathrm{~h}$ until $4.5 \mathrm{~h}$, after which the medium was switched back to standard 7H9 OADC medium. Up to $80 \mathrm{XY}$ points were imaged at 10-minute intervals on fluorescence and phase channels for up to $36 \mathrm{~h}$. The

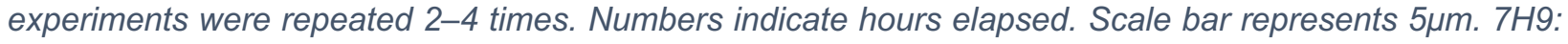
Middlebrook 7H9 medium. MMC: Mitomycin C. 


\section{Purified mutasome proteins interact in biochemical assays in vitro.}

224 All inference from this and previous work about the composition of the mycobacterial mutasome has 225 been derived from microbiological assays. To address this limitation, we expressed and purified 226 recombinant M. smegmatis mutasome proteins for biochemical analysis. Expression in E. coli of ImuB 227 alone yielded low quantities of soluble protein that was prone to degradation, while attempts to 228 express ImuA' alone failed to generate soluble protein. In contrast, co-expression of ImuB with ImuA' yielded both proteins in a soluble form (Figure 3). Subsequently, the ImuA'B complex could be captured via a histidine (His) affinity tag in ImuB. This confirmed that ImuA' and ImuB interact in vitro, forming a stable complex even at protein concentrations as low as $400 \mathrm{nM}$ (Figure 3 - figure supplement 1), corroborating previous yeast two-hybrid results (Warner et al., 2010). In E. coli, overexpression of DnaE2 resulted in insoluble protein, while DnaE2 overexpression in M. smegmatis appeared to be incompatible with cell viability: following transformation with the expression construct, very few colonies were obtained and could not be expanded in liquid culture (not shown). Next, we analysed the interaction of the $d n a N$-encoded $\beta$ clamp with ImuB or the ImuA ${ }^{\prime} B$ complex (Figure 3). Samples of the M. smegmatis $\beta$ clamp with ImuA'B (Figure 3A panel i) or ImuB (Figure 3A panel ii), were injected onto an analytical size-exclusion chromatography column and collected fractions were analysed by SDS-PAGE. Alone, the $\beta$ clamp and ImuB/ImuA'B eluted at 1.47 and $1.55 \mathrm{ml}$, respectively. Incubation of the $\beta$ clamp with either ImuB or ImuA'B caused a shift in the retention volume to $1.36 \mathrm{ml}$, indicative of complex formation. This was confirmed by SDS-PAGE analysis, which indicated co-elution of the $\beta$ clamp with ImuB and ImuA'B.

-ImuB and VFP-ImuA' form a stable complex.

244 Our microbiological assays had unexpectedly revealed discrepant complementation phenotypes for 245 UV exposure (Figure 1 - figure supplement 2A) versus MMC treatment (Figure 1 - figure supplement $2 B$ ), raising the possibility that the fluorescent tags in the bioreporter mutants might disrupt a protein-protein interaction(s) essential for DNA damage tolerance. We therefore investigated the capacity of the fluorescently labelled EGFP-ImuB and VFP-ImuA' proteins to form a stable complex. To this end, His-EGFP-ImuB was co-expressed with Strep-VFP-ImuA' in E. coli and the complex analysed in three consecutive chromatography steps (Figure 3 - figure supplement

251 1B). First, the cell lysate was loaded onto a HisTrap column to capture the VFP-ImuA':EGFP-ImuB 252 complex via the His-tag present in EGFP-ImuB. Next, the elution fractions containing the complex 253 were loaded on a StrepTrap column to capture the complex via the strep-tag on VFP-ImuA'. Finally, 254 the VFP-ImuA':EGFP-ImuB complex was injected onto a size-exclusion column. 
A

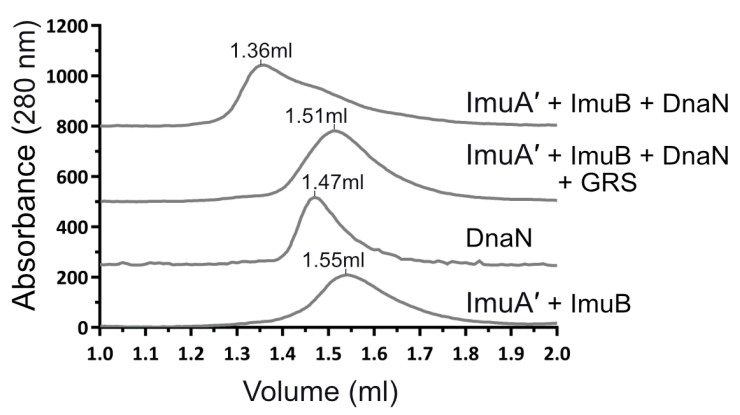

B

ImuA'
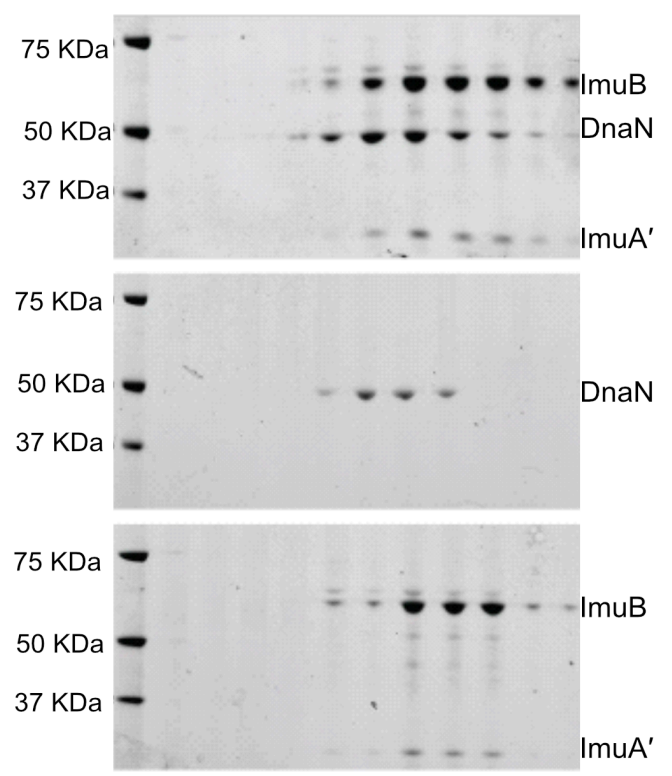
filtration profiles shown in A.

ii

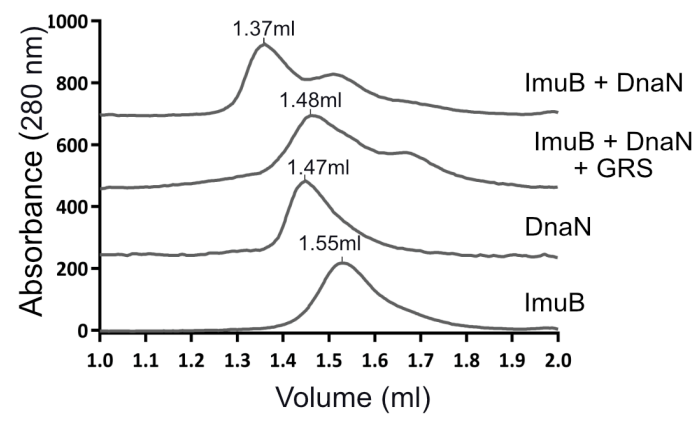

ii
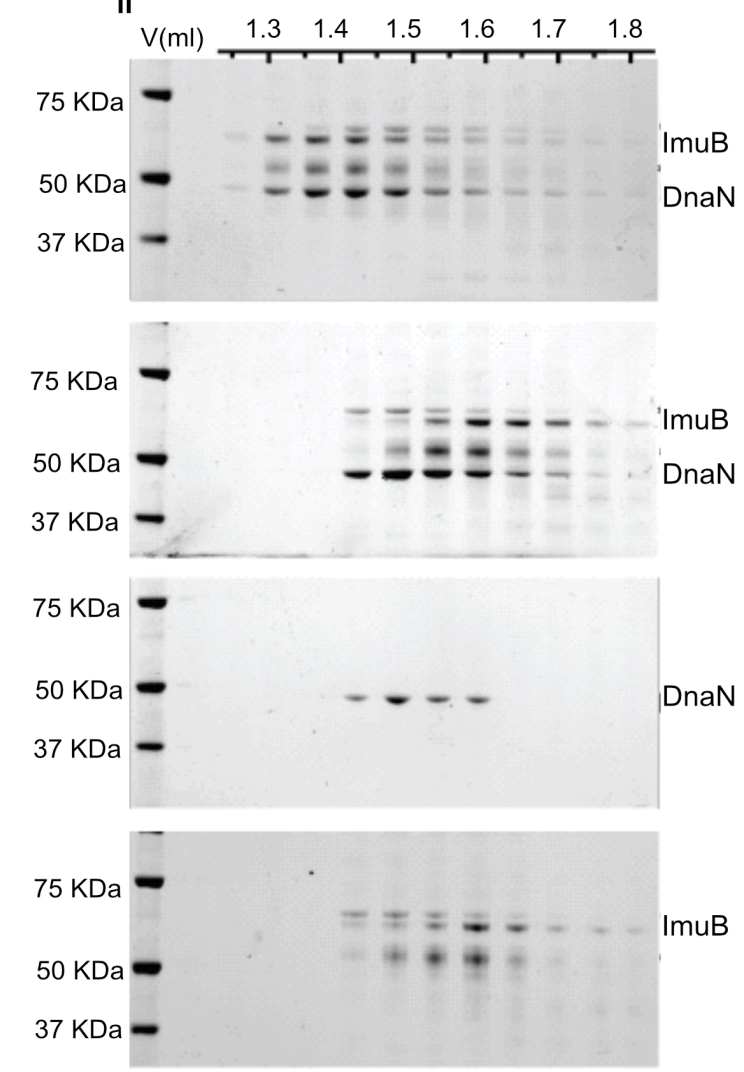

Figure 3. ImuB and ImuA'-ImuB interact with the DnaN and these interactions are disrupted by GRS.

A. Gel filtration profiles of M. smegmatis (i) ImuA'-ImuB-DnaN and (ii) ImuB-DnaN complexes in the absence or presence of $15 \mu \mathrm{M}$ GRS. For these experiments, $5 \mu \mathrm{M}$ DnaN was added to $10 \mu \mathrm{M}$ of (i) ImuA'ImuB or (ii) ImuB. The gel filtration profiles of the individual proteins (ImuB and DnaN) or complex (ImuA'ImuB) are shown for comparative purposes, and all curves were scaled for clarity. B. SDS-PAGE analysis of sequential fractions of the gel filtration runs. Gels are sorted in the same order as the corresponding gel 
263 During all purification steps, EGFP-ImuB with VFP-ImuA' were co-eluted as a complex, as indicated

264 by SDS-PAGE analysis and fluorescent detection of GFP-ImuB and VFP-ImuA' in the same elution

265 fractions. In combination, these observations suggest that the fluorescent tags did not disrupt ImuA'-

266 ImuB complex formation - a result which additionally implies that the absence in live cells of a clear

267 ImuA' (co-)localization phenotype was not a function of the tags.

268 Inhibition of ImuB- $\beta$ clamp binding eliminates focus formation.

269 Previous work established that the $\beta$ clamp-binding domain of ImuB was essential for mutasome

270 function: mutant strains carrying either a $i m u B^{\Delta}{ }^{C 168}$ allele - which lacks the 168 amino acids in the

271 ImuB C-terminal region - or a imuBAAAAGG allele - in which the wild-type $\beta$ clamp-binding motif,

$272{ }^{352} \mathrm{QLPLWG}^{357}$, is substituted with the non-functional ${ }^{352} \mathrm{AAAAGG}^{357}$ peptide sequence - phenocopied

273 full $i m u B$ deletion (Warner et al., 2010). Therefore, to test the prediction that the SOS-regulated

274 recruitment of EGFP-ImuB and mCherry- $\beta$ clamp into discernible foci was dependent on the ImuB- $\beta$

275 protein-protein interaction, we introduced an egfp-imuBAAAAGG allele into the $\triangle i m u B$ mutant. In

276 contrast to the wild-type reporter (G-imuB), the $\beta$ clamp-binding motif mutant (G-imuBAAAGG)

277 exhibited no EGFP foci in any cell imaged following exposure to MMC (Figure 4A panel ii). Instead,

278 the fluorescence was detectable throughout the cell as a diffuse signal (Figure 4B). This result

279 supports the inferred essentiality of the physical interaction between ImuB and $\beta$ for ImuB

280 localization and, moreover, establishes that detection of ImuB- $\beta$ foci provides a reliable visual proxy

281 for functional mutasome formation.

\section{Griselimycin blocks ImuB- $\beta$ clamp binding, preventing focus formation in $\boldsymbol{M}$. smegmatis.}

283 Griselimycin (GRS) is a natural product antibiotic that binds the mycobacterial $\beta$ clamp with high

284 affinity, preventing DNA replication by blocking the essential interaction with the PolIII $\alpha$ subunit,

285 DnaE1 (Kling et al., 2015). Notably, the location of GRS binding overlaps with the region predicted to

286 interact with other $\beta$ clamp-binding proteins, including ImuB (Bunting et al., 2003; Burnouf et al.,

287 2004; Kling et al., 2015). Therefore, we hypothesized that GRS might disrupt the ImuB- $\beta$ interaction.

288 Indeed, addition of GRS disrupted the in vitro interaction between the $\beta$ clamp and pre-formed

289 ImuA'B complex (Figure 3A panel i) as well as between the $\beta$ clamp and ImuB (Figure 3A panel ii),

290 as indicated by a gel filtration profile that is a superposition of the absorbance traces of the sample

291 individual components ( $\beta$ clamp and ImuB or $\beta$ clamp and ImuA'B). This was confirmed by SDS-PAGE

292 analysis (Figure 3 B). To confirm that the disrupting effect of GRS on the complex was the result of

293 the GRS- $\beta$ clamp binding (Kling et al., 2015), we measured the melting curves of the $\beta$ clamp in the

294 presence and absence of GRS (Figure 3 - figure supplement 1C). Incubation with GRS led to a $3{ }^{\circ} \mathrm{C}$ 
bioRxiv preprint doi: https://doi.org/10.1101/2021.11.16.468908; this version posted November 17, 2021. The copyright holder for this preprint (which was not certified by peer review) is the author/funder, who has granted bioRxiv a license to display the preprint in perpetuity. It is made available under aCC-BY-ND 4.0 International license. of GRS was observed on the melting temperatures of ImuA'B.
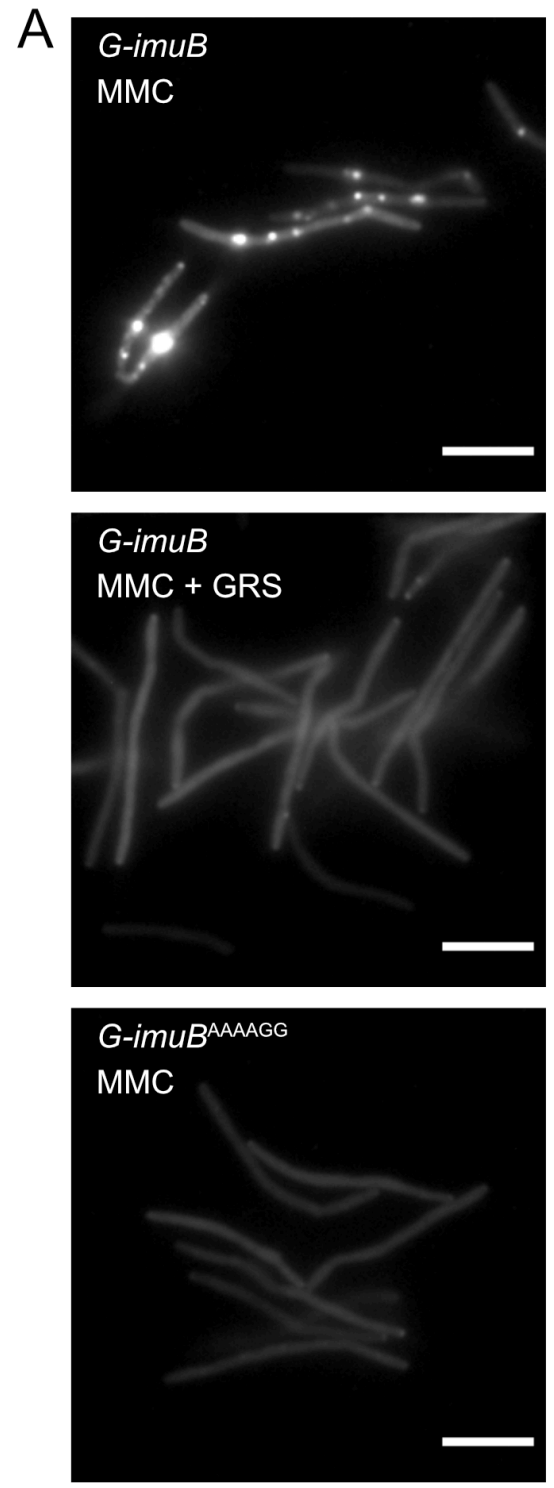

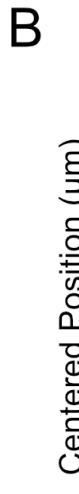

Wild-type G-imuB G-imuB $B^{\text {AaAgG }}$
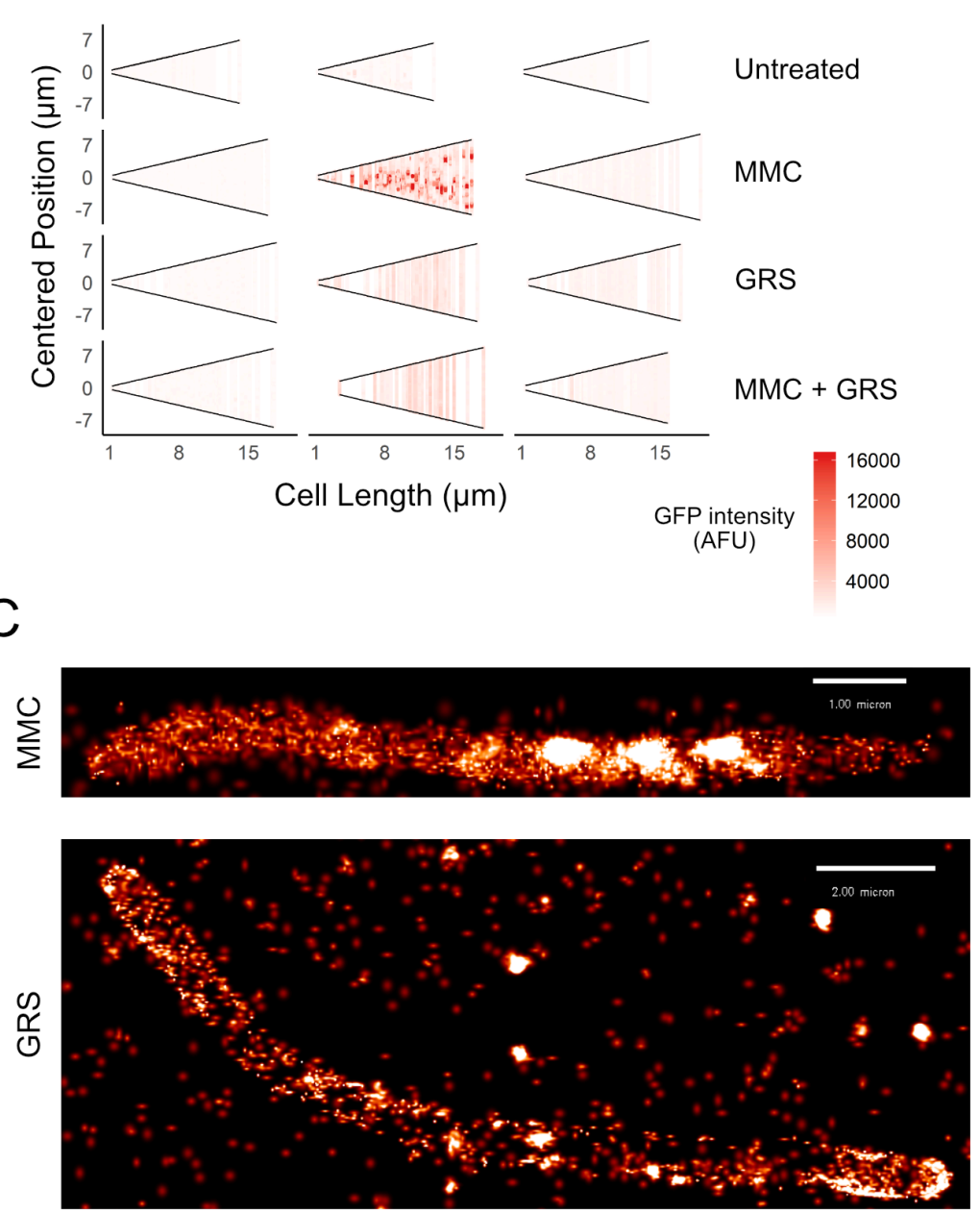

Molecule probability

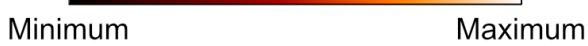

Figure 4. Disrupting the DnaN-ImuB interaction. A. Representative images of G-imuB exposed to $2 \times$ MIC MMC for $4 \mathrm{hr}$ (top panel) or 2× MIC MMC and GRS for $4 \mathrm{hr}$ (centre panel) and G-imuB ${ }^{A A A A G G}$ mutant exposed to 2× MIC MMC for $4 \mathrm{hr}$ (bottom panel). Scale bars represent $5 \mu \mathrm{m}$. B. Cells aligned by mid-cell position, arranged according to cell length and coloured (white to red) according to fluorescence intensity showing the presence of G-ImuB foci following MMC treatment and the lack thereof after GRS treatment. G-imuB ${ }^{A A A A G G}$ shows no foci, similar to the G-imuB strain following GRS exposure. C. Super-resolution imaging confirms disruption of ImuB- $\beta$ clamp foci in mycobacteria treated with GRS. Representative iPALM localizations of PC-ImuB bacilli exposed to (top) $5 \times$ MIC MMC or (bottom) $5 \times$ MIC GRS. Subdiffractionlimited super-resolution localization of PC-ImuB is observed as highly dense localizations of signal following exposure to MMC; in contrast, GRS prevents the formation of high-density localizations. Scale bars are 1 $\mu \mathrm{m}$ and $2 \mu \mathrm{m}$ in the top and bottom micrographs, respectively; molecule probability represents the fluorescence localization probability from minimum (black) to maximum (white) likelihood. 
Next, we examined whether these in vitro observations were recapitulated in live mycobacterial cells. To this end, the G-imuB strain was exposed to MMC (Figure $4 \boldsymbol{A}$ panel i) or MMC

312 plus GRS (Figure 4A panel iii). Notably, the addition of GRS in combination with MMC abrogated G-

$313 i m u B$ focus formation, phenocopying the diffuse fluorescence distribution observed following 314 exposure of the $\beta$ clamp-binding deficient GFP-ImuBAAAAGG mutant to MMC Figure 4 A panel ii).

315 Population analyses of these data confirmed that GRS blocked ImuB focus formation in MMC-exposed

316 cells (Figure 4 B), suggesting the potential for chemical disruption of mutasome function.

317 Super-resolution microscopy confirms GRS elimination of ImuB- $\beta$ foci in M. smegmatis.

318 Finally, to examine the inferred disruption of ImuB focus formation by single-molecule microscopy, 319 we generated an additional reporter mutant in which ImuB was N-terminally labeled with the 320 photoconvertible, fixation-resistant mEos4a fluorophore (Paez Segala et al., 2015). The resulting PC$321 i m u B$ strain was imaged in 3D by iPALM (Shtengel et al., 2009) following exposure to MMC or GRS

322 (Figure 4C). Consistent with the epifluorescence data, bacilli exposed to MMC were characterized by 323 a region of high-density PC-ImuB localizations (Figure 4C top panel). In contrast, exposure to GRS 324 which on its own has been shown to induce the M. smegmatis SOS response (Kling et al., 2015) - did 325 not elicit high molecule densities: instead, a low-density of molecules was detected throughout the 326 interior of the cell, reinforcing the inferred absence of ImuB recruitment in GRS-exposed cells (Figure 4C bottom panel).

\section{DISCUSSION}

In E. coli, the DNA damage-induced SOS response triggers overexpression of $u m u C$, umuD and recA

330 (Maslowska et al., 2019). UmuC is an error prone Y-family DNA polymerase that requires the binding 331 of $\mathrm{UmuD}_{2}$ and RecA to reach full activity and this "mutasome", collectively referred to as PolV, has 332 been implicated in DNA damage tolerance and induced mutagenesis (Goodman et al., 2016). At the 333 onset of the work reported here, genetic evidence from diverse bacteria lacking PolV supported the 334 co-dependent operation of ImuA, ImuB, and DnaE2 in the LexA-regulated SOS response, suggesting 335 these proteins might function in an analogous manner (McHenry, 2011; Ippoliti et al., 2012). In 336 mycobacteria, in which they have also been implicated in DNA damage tolerance and induced 337 mutagenesis (Boshoff and Mizrahi, 2000; Warner et al., 2010), the non-homologous ImuA' replaces 338 ImuA, nevertheless the inferred universal model for mutasome function in bacteria lacking an E. coli 339 PolV homolog was the same (Timinskas and Venclovas, 2019): the catalytically inactive Y family 340 polymerase, ImuB, functions as hub protein, interacting physically with the $\beta$ clamp via a defined $\beta$ 341 clamp-binding motif and with DnaE2 and ImuA' (or ImuA) via unknown mechanisms which might 
include the disordered ImuB C-terminal region or sub-regions thereof, including the RecA-NT motif (Timinskas and Venclovas, 2019). However, the absence of any direct biochemical and/or structural evidence to support the proposed protein interactions meant this assumption was speculative. Moreover, whereas E. coli PolV is known to be subject to multiple forms of regulation - including temporal (Robinson et al., 2015), spatial (Robinson et al., 2015), internal (Erdem et al., 2014) and conformational (Jiang et al., 2009; Gruber et al., 2015; Jaszczur et al., 2019) - the expression dynamics and sub-cellular localizations of the mycobacterial mutasome proteins were mostly unknown.

By fluorescently tagging the known mutasome proteins, we have observed in real-time the consistent formation of co-localizing ImuB- $\beta$ clamp foci in mycobacterial cell populations exposed to genotoxic stress. Although less pronounced than ImuB, we also detected the frequent, reproducible co-localization of DnaE2 with the $\beta$ clamp under the same conditions. Notably, recruitment of ImuB into foci occurred in mutants lacking either $d n a E 2$ or $i m u A^{\prime}$ but was prevented where the ImuB $\beta$ clamp-binding motif was mutated - apparently identifying the primacy of the ImuB- $\beta$ clamp interaction in mutasome organization. In contrast, the function(s) and sub-cellular dynamics of ImuA' remain enigmatic: VFP-ImuA' consistently produced diffuse fluorescence in DNA-damaged bacilli, precluding any definitive insights into its potential association with ImuB (or DnaE2) in vivo. We did, however, observe co-elution of ImuA'-ImuB and ImuA'-ImuB- $\beta$ clamp complexes in vitro, results which provided important biochemical confirmation of the inferred interaction of ImuA ${ }^{\prime}$ and ImuB predicted previously (Warner et al., 2010). While difficult to reconcile with these data, the absence of a clear co-localization signal in live cells might indicate the transient association of $\operatorname{ImuA}^{\prime}$ with its mutasome partners, or possibly a modification analogous to the proteolytic cleavage of UmuD to UmuD' in the E. coli SOS response (Goodman et al., 2016). Future work will require single-molecule tracking of ImuA' to resolve this possibility.

The original identification of the imuA-imuB-dnaE2 cassette noted its close association with LexA across diverse bacteria; that is, genomes containing the cassette invariably encoded a LexA homolog, too (Erill et al., 2006). Recent work in mycobacteria has, however, added unexpected nuance to that regulatory framework, namely that the split $i m u A^{\prime}-i m u B / d n a E 2$ cassette is subject to transcriptional control by both the "classic" LexA/RecA-regulated SOS response and the PafBCmediated DNA damage response. The authors also report that, while the two regulatory mechanisms are partially redundant for genotoxic stresses including UV and MMC exposure, fluoroquinolones appear to be specific inducers of PafBC only (Adefisayo et al., 2021). In addition to suggesting that chromosomal mutagenesis is co-dependent on PafBC and SOS, these observations are important in identifying an apparent "fail-safe" mechanism in mycobacteria in which the mutasome components 
375 are induced irrespective of DNA damage type - again reinforcing the centrality of these proteins in damage tolerance and, by implication, adaptive mutagenesis.

We previously observed that the imuBAAAAGG $\beta$ clamp-binding mutation eliminated UVinduced mutagenesis and MMC damage tolerance in M. smegmatis (Warner et al., 2010), phenocopying deletion of any of the three mutasome components $\left(i m u A^{\prime}\right.$, imuB, dnaE2) alone or in combination. Given the abrogation of ImuB focus formation, it is reasonable to infer a direct link between ImuB- $\beta$ clamp focus formation and mutasome function. In turn, this suggests that blockade of ImuB focus formation might offer a tractable read-out for a screen designed to identify mutasome inhibitors - a possibility reinforced by the observed co-elution in biochemical assays of $\beta$ with ImuB and, separately, of the $\beta$ clamp with pre-formed ImuA'-ImuB complexes. In this context, it was notable in the current work that GRS disrupted the ImuB- $\beta$ clamp interaction in vitro and prevented ImuB focus formation in mycobacteria treated simultaneously with MMC and GRS.

The discrepant complementation phenotypes observed for V-imu $A^{\prime}$ and G-imuB in the DNA damage tolerance (MMC treatment) versus induced mutagenesis (UV exposure) assays suggests that addition of the bulky fluorophore might have prevented full function of these mutasome proteins. Whereas UV irradiation predominantly generates cyclobutane dimers and pyrimidine-pyrimidone (6-4) photoproducts (Franklin et al., 1985), MMC induces different DNA lesions, including inter- and intra-strand crosslinks. These are likely to require multiple repair proteins and, potentially, the interaction of mutasome components with additional proteins - which might be prevented by the bulky fluorescent tags. The DnaE2-EGFP fusion proved the exception; in this context, it might be instructive to consider recent evidence implicating DnaE2 in gap filling following nucleotide excision repair in non-replicating Caulobacter crescentus cells (Joseph et al., 2021). These observations suggest the importance of identifying other potential interacting partners of mycobacterial DnaE2 (and the other mutasome components), work which is currently underway in our laboratory.

The potential for inhibitors of DNA replication to accelerate the development of genetic resistance through the induction of mutagenic repair pathways (Cirz et al., 2005; Barrett et al., 2019; Revitt-Mills and Robinson, 2020) is a valid and commonly cited concern that might partially explain the relative under-exploration of DNA metabolism as source of new antibacterial drug targets (Reiche et al., 2017; van Eijk et al., 2017). Our results suggest that GRS could offer an interesting exception: that is, in binding the $\beta$ clamp at the site of interaction with the DnaE1 replicative DNA polymerase (Kling et al., 2015) as well as other DNA metabolizing proteins, including ImuB, GRS appears to possess an intrinsic protective mechanism against induced mutagenesis - blocking both ImuB-dependent mutasome recruitment to stalled replisomes and post-repair fixation of mutations 
by the replicative polymerase, DnaE1. This "resistance-proofing" capacity, which is supported by the reported restriction of GRS resistance to low-frequency, high-fitness cost amplifications of the dnaN genomic region with very few to no "off-target" SNPs, might also contribute to the observed bactericidal effect of GRS against mycobacteria (Kling et al., 2015). In addition, it reinforces the $\beta$ clamp as vulnerable target for new TB drug development (Bosch et al., 2021). In this context, it is worth noting that inhibition of DnaE1 replicative polymerase function might represent a general solution to the problem of drug-induced (auto)mutagenesis by preventing fixation of repair/tolerance-generated mutations; in support of this inference, another natural product, nargenicin, which inhibits $M t b$ DnaE1 via a DNA-dependent mechanism, fails to yield spontaneous resistance mutations in vitro (Chengalroyen et al., 2021). Therefore, while the essentiality of DNA replication proteins (including DnaN, DnaE1) for mycobacterial viability poses a challenge to the design of assays of "anti-evolution" compounds targeting these proteins, GRS (and nargenicin) appear to provide compelling evidence that precise inhibition of specific DNA replicative and repair functions might ameliorate the perceived risks in targeting this area of mycobacterial metabolism.

As an obligate human pathogen, persistence of M. tuberculosis within its host depends on the ability to drive successive cycles of infection, disease - in some cases latency followed by reactivation disease - and transmission (Lin and Flynn, 2018). This process is necessarily vulnerable to multiple potential evolutionary culs-de-sac which might arise in consequence of the elimination of the bacillus by the host (clearance) or the demise of the organism within the infected individual (controlled subclinical infection, or host death). Modern M. tuberculosis strains therefore represent the 428 genotypes that have successfully adapted to human colonization (Gagneux, 2018), evolving with their obligate host through changes in lifestyle and nutritional habits (with their associated implications

430 for non-communicable diseases such as diabetes), the near-universal administration of the BCG 431 vaccination, the emergence of the HIV co-pandemic, and the widespread use of frontline combination 432 chemotherapy (Warner et al., 2015). While the emergence and propagation of drug-resistant isolates 433 characterized by a variety of polymorphisms at multiple genomic loci (Warner et al., 2017; Farhat et 434 al., 2019; Payne et al., 2019) provides strongest proof of the capacity for genetic variation in $M$. 435 tuberculosis, other lines of evidence include the highly subdivided population structure of the $M$. 436 tuberculosis Complex (Riojas et al., 2018), the well-described geographical host-pathogen sympatry 437 (Hershberg et al., 2008; Brynildsrud et al., 2018) and, more recently, the observation of intra-patient 438 bacillary microdiversity (Ley et al., 2019). In combination, these elements support the ongoing 439 evolution of $M$. tuberculosis, as well as suggest the potential that "anti-evolution" therapeutics might 440 yield much greater benefit in the clinical context than can be inferred from in vitro studies - in which 
441 the pressures on an obligate pathogen can only be approximated. That is, in addition to identifying

442 the mutasome as target for adjunctive therapeutics designed to protect anti-TB drugs against

443 emergent resistance, the results presented here support the further exploration of this and related

444 strategies to disarm host-adaptive mechanisms in a major human pathogen.

\section{MATERIALS AND METHODS}

\section{Bacterial strains and culture conditions}

447 All mycobacterial strains (Supplementary File 2 - Key Reagents) were grown in liquid culture 448 containing Difco ${ }^{\mathrm{TM}}$ Middlebrook 7H9 Broth (BD Biosciences, San Jose, CA) and supplemented with 0.2 $449 \%$ (v/v) glycerol (Sigma Aldrich, St. Louis, MO), 0.005 \% (v/v) Tween® 80 (Sigma Aldrich, St. Louis, $450 \mathrm{MO}$ ), and $10 \%$ (v/v) BBL ${ }^{\mathrm{TM}}$ Middlebrook OADC Enrichment (BD Biosciences, San Jose, CA). For $M$. 451 smegmatis, liquid cultures were incubated at $37^{\circ} \mathrm{C}$ with orbital shaking at $100 \mathrm{rpm}$, until the desired 452 growth density was attained - measured by spectrophotometry at a wavelength of $600 \mathrm{~nm}$ - before 453 further experimentation. Solid media comprised Difco ${ }^{\mathrm{TM}}$ Middlebrook 7H10 Agar (BD Biosciences, 454 San Jose, CA) supplemented with $0.5 \%$ (v/v) glycerol (Sigma Aldrich, St. Louis, MO), and 10 \% (v/v) 455 BBL ${ }^{\text {TM }}$ Middlebrook OADC Enrichment (BD Biosciences, San Jose, CA). Solid media plates were 456 incubated at $37^{\circ} \mathrm{C}$ for 3-4 days or until colonies had formed.

\section{Mutasome reporter constructs}

458 The V-imuA' construct was designed by altering the coding sequence of imu $A^{\prime}$ within the 459 complementing vector, pAINT::imuA' (Warner et al., 2010), so that the coding sequence of Venus 460 fluorescent protein (VFP) (Nagai et al., 2002) was inserted in-frame after the start codon of the $i m u A^{\prime}$ 461 ORF. Furthermore, an in-frame FLAG tag sequence (Einhauer and Jungbauer, 2001) was inserted 462 between the coding region of $v f p$ and $i m u A^{\prime}$ to produce a single ORF encoding VFP-FLAG-ImuA'. For 463 ImuB, the construct PSOS(imuA)-egfp-imuB was designed such that the regulatory elements 464 immediately upstream of $i m u A^{\prime}$ were inserted immediately upstream of the $i m u B$ ORF which was 465 further altered by inserting the sequence encoding EGFP (Cormack et al., 1996) linked to a FLAG tag466 encoded sequence immediately after the start codon of $i m u B$ to produce a single ORF encoding EGFP467 FLAG-ImuB' which was cloned into pMCAINT::imuB (Warner et al., 2010). The photoconvertible 468 PSOS(imuA')-mEos4A-imuB construct was based on the G-imuB construct, such that the coding 469 sequence of EGFP was replaced by mEos4a (Paez Segala et al., 2015), yielding mEos4A-FLAG-ImuB. 470 For DnaE2, the egfp sequence was inserted in-frame after the start codon of M. smegmatis dnaE2.

471 Mutant binding G-imuB ${ }^{\text {AAAAGG }}$ construct 
472 To introduce the ${ }^{352} \mathrm{AAAAGG}^{357}$ ImuB allele (Warner et al., 2010) into the EGFP-ImuB protein, the

473 nucleotide sequence from pMCAINT::imuBAAAGG was swapped into the corresponding position of

474 PSOS(imuA')-egfp-imuB to yield pMCAINT::PSOS(imuA')-egfp-imuBAAAAGG.

\section{M. smegmatis mutasome reporter strains}

476 M. smegmatis strain $\mathrm{V}$-imu $A^{\prime}$ was generated by introducing the pAINT::vfp-imuA' plasmid into $\Delta i m u A^{\prime}$

477 (Warner et al., 2010) by the standard electroporation method. Strains G-imuB, PC-imuB, and G478 imuBAAAAGG were developed by integration of the pMCAINT::PSOS(imuA)-egfp-imuB, 479 pMCAINT::PSOS(imuA)-mEos4a-imuB, or pMCAINT::PSOS(imuA)-egfp-imuBAAAAG plasmid,

480 respectively, into the genome of $\triangle i m u B$ (Warner et al., 2010). The dnaN-mCherry::G-imuB strain was 481 developed by the electroporation of pMCAINT::PSOS(imuA')-egfp-imuB into the M. smegmatis dnaN482 mCherry background (Santi et al., 2013). To generate the G-dnaE2 strain, pTweety::egfp-dnaE2 was 483 electroporated into $\Delta d n a E 2$ (Warner et al., 2010). Similarly, dnaN-mCherry::G-dnaE2 was generated 484 by electroporation of pTweety::egfp-dnaE2 into M. smegmatis dnaN-mCherry. Mutasome-deficient 485 strains $\triangle i m u A^{\prime}, \Delta d n a E 2$, and dnaE2 ${ }^{A I A}$ were electroporated with pMCAINT::PSOS(imuA')-egfp-imuB 486 to produce $\Delta i m u A^{\prime}:: G$-imuB, $\triangle d n a E 2:: G$-imuB, and dnaE2AIA::G-imuB, respectively.

\section{Mutagenesis Assays}

488 Mutagenesis assays were performed as previously described (Boshoff et al., 2003; Warner et al., 489 2010), with RIF-resistant colonies enumerated on solid media after 5 days of growth. Mutation

490 frequencies were calculated by dividing the number of RIF-resistant colonies of each sample by the $491 \mathrm{CFU} / \mathrm{ml}$ of un-irradiated sample.

\section{Antibiotic treatments}

493 MMC (Mitomycin C from Streptomyces caespitosus) (Sigma Aldrich, St. Louis, M0) was dissolved in $494 \mathrm{ddH}_{2} \mathrm{O}$, while GRS was dissolved in DMSO. Cultures of M. smegmatis were grown in 7H9-OADC495 supplemented with selection antibiotic where applicable-at $37^{\circ} \mathrm{C}$ to an optical density $\left(\mathrm{OD}_{600}\right){ }^{\circ}$ of 496 between 0.2 and 0.4 . Thereafter, cultures were split into different $5 \mathrm{ml}$ cultures and MMC and/or GRS 497 was added to a final concentration dependent on the MIC (Kling et al., 2015).

\section{Snapshot microscopy}

499 Single snapshot micrographs of M. smegmatis cells were captured with a Zeiss Axioskop M, Zeiss 500 Axio.Scope, and Zeiss Axio.Observer Z1. Briefly, 2.0-5.0 $\mu \mathrm{l}$ of liquid culture was placed between a No. $501 \quad 1.5$ glass coverslip and microscope slide. A transmitted mercury lamp light was used together with 502 filter cubes to visualize fluorescence using a 100× 1.4 NA plan apochromatic oil immersion objective 503 lens. Samples were located using either transmitted light, differential interference contrast (DIC), or 504 epifluorescence. Snapshot images were captured with either a Zeiss 1 MP or Zeiss AxioCam HRm 
monochrome camera. Images of the same experiment were captured with the same instrument and exposure settings. Green fluorescence of EGFP was detected using the Zeiss Filter Set 38 HE. Red

507 fluorescence of mCherry was detected using the Zeiss Filter Set 43. Images were captured using 508 AxioVision 4.7 or ZEN Blue Microscope and Imaging Software. Images were processed using Fiji 509 (Schindelin et al., 2012); images of the same strain were contrasted to the same maximum and 510 minimum within an experiment.

\section{Quantitative image analysis}

512 M. smegmatis bacilli were plotted from shortest to longest and aligned according to their midcell 513 position ( 0 on the y axis) using the MicrobeJ plugin of ImageJ (Ducret et al., 2016). Along each point 514 of the cell, a dot was generated and coloured according to the fluorescence intensity along the medial 515 axis of the bacillus. Therefore, this plot represents the fluorescence intensity along the medial axis of 516 every bacillus imaged under the relevant experimental conditions. $\mathrm{R}$ was used for visual 517 representation of the data.

\section{Super-resolution iPALM microscopy}

519 Three-dimensional PALM was performed using the iPALM instrument (Shtengel et al., 2009). Round $25 \mathrm{~mm}$ diameter No. 1.5 coverslips were cleaned by sonication in $1 \mathrm{M} \mathrm{KOH}$ for 45 minutes. Following rinsing in deionized water and drying at $60{ }^{\circ} \mathrm{C}$, coverslips were coated with $5 \mathrm{mg} / \mathrm{ml}>70,000$ molecular weight poly-L-lysine hydrobromide (MP Biomedicals, Santa Ana, CA) for 30 minutes at room temperature. Thereafter, gold nanorods were adhered to the coverslips for 30 minutes before drying by vacuum centrifugation. Thin film deposition was used to coat the fiducial coverslips with $\mathrm{SiO}_{2}$. Thereafter, the fiducial coverslips were cleaned with $1 \mathrm{M} \mathrm{KOH}$ and coated with $1 \%$ poly-L-lysine for 60 minutes at $37.0^{\circ} \mathrm{C}$. Bacterial cultures of $\mathrm{OD}_{600}=0.4$ were exposed to drug conditions $(5 \times \mathrm{MMC}$ or GRS) for $6 \mathrm{~h}$ before $3.0 \mathrm{ml}$ of bacterial sample was centrifuged onto a fiducial coverslip at 3,200 rcf for 15 minutes in a six-well plate. The sample was rinsed three times in Dulbecco's PBS and fixed with $0.5 \%$ paraformaldehyde for 2 minutes. Thereafter, the sample was mounted in Dulbecco's PBS and the gold coverslip were adhered to a clean (as above) 18mm diameter No. 1.5 round coverslip. Each coverslip was sealed to prevent evaporation. The sample was mounted between two opposing Nikon 60× 1.49 NA Apo TIRF oil immersion lenses and captured using three Andor iXon-3 EMCCD cameras. Bacterial cells were located by DIC visualization, and each sample was imaged three times at separate regions containing 2-5 bacilli using TIRF illumination. Experiments were repeated at least three times. A calibration image of 100 cycles was taken of the gold fiducials in each field-ofview. Bacilli were imaged for 25,000 cycles using alternating $405 \mathrm{~nm}$ (mEos4a photoconversion) and $561 \mathrm{~nm}$ (converted mEos4a excitation) laser cycles per frame. FF01-593/40 emission filters 
538 (Semrock) were used during mEos4a imaging. Thereafter, the calibration file was processed using

539 PeakSelector ${ }^{\mathrm{TM}}$ (Shtengel et al., 2009) and used to calibrate the detected localizations. During image

540 processing, low confidence localizations were excluded based on unwrapped Z-error and Z-position.

541 Images were produced with PeakSelector ${ }^{\mathrm{TM}}$.

\section{Single-cell time-lapse fluorescence microscopy}

543 Liquid cultures of $M$. smegmatis reporter strains were grown to mid-logarithmic phase $\left(\mathrm{OD}_{600}=0.6\right)$,

544 cells were collected by centrifugation at $3900 \times g$ for 5 min and concentrated 10 -fold in 7 H9 medium.

545 The cells were filtered through a polyvinylidene difluoride (PVDF) syringe filter (Millipore) with a 5

$546 \mu \mathrm{m}$ pore size to yield a clump-free cell suspension. The single cell suspension was spread on a semi-

547 permeable membrane and secured between a glass coverslip and the serpentine 2 chip (Delincé et al.,

548 2016) in a custom-made PMMA/Aluminium holder (Dhar and Manina, 2015). Time-lapse microscopy

549 employing a DeltaVision personalDV inverted fluorescence microscope (Applied Precision, WA) with

550 a 100x oil immersion objective was used to image single cells of $M$. smegmatis. The bacteria and

551 microfluidic chip were maintained at $37{ }^{\circ} \mathrm{C}$ in an environmental chamber with a continuous flow of

$5527 \mathrm{H} 9$ medium, with or without $100 \mathrm{ng} / \mathrm{ml}$ of MMC, at a constant flow rate of $25 \mu \mathrm{l} . \mathrm{min}^{-1}$, as described

553 previously (Wakamoto et al., 2013; Dhar and Manina, 2015). Images were obtained every 10 min on

554 phase-contrast and fluorescence channels (for EGFP, excitation filter 470/40 nm, emission filter

555 525/50 nm; for mCherry, excitation filter 572/35, emission filter 632/60; for YFP excitation filter

$556500 / 20 \mathrm{~nm}$, emission filter 535/30 nm) using a CoolSnap HQ2 camera. Image-based autofocus was

557 performed on each point prior to image acquisition. Experiments were repeated 2-4 times; a typical

558 experiment collected images from up to $80 \mathrm{XY}$ points at the $10 \mathrm{~min}$ intervals. The images were

559 analysed using Fiji (Schindelin et al., 2012).

560 Protein expression and purification

561 N-terminally His-tagged M. smegmatis ImuB was co-expressed with ImuA' in E. coli BL21(DE3) cells

562 using two expression vectors from the NKI-LIC vector suite (Luna-Vargas et al., 2011): pETNKI-his-

563 3C-LIC-kan for ImuB and pCDFNKI-StrepII3C-LIC-strep for ImuA' that have different resistance

564 markers, kanamycin and streptomycin; as well as different origins of replication, ColE1 and CloDF13,

565 respectively. Protein production was induced with isopropyl 1-thio- $\beta$-d-galactopyranoside (IPTG) at

$56630^{\circ} \mathrm{C}$ for 2 hours. The ImuBA' complex was purified using a Histrap column followed by a Superdex

567200 16/60 column. Both N-His6 M. smegmatis ImuB and $\beta$ clamp were expressed in E. coli BL21(DE3)

568 cells and purified using HisTrap, HiTrap Q, and S200 columns. All proteins were flash frozen in liquid

569 nitrogen and stored at $-80{ }^{\circ} \mathrm{C}$.

570 Size-exclusion chromatography analysis 
571 Samples of individual proteins and the different complexes were injected onto a PC3.2/30 (2.4 ml)

572 Superdex 200 Increase gel filtration column (GE Healthcare) pre-equilibrated in $50 \mathrm{mM}$ Tris pH 8.5

573 and $300 \mathrm{mM} \mathrm{NaCl}$. Thereafter, $50 \mu$ fractions were collected and analyzed by SDS-PAGE

574 electrophoresis using 4-12\% NuPage Bis-Tris precast gels (Life Technologies). Gels were stained

575 with $0.01 \%(\mathrm{v} / \mathrm{v})$ 2,2,2-Trichloroethanol (TCE) and imaged with UV light.

\section{Thermal unfolding experiments}

577 Melting curves of the M. smegmatis $\beta$ clamp $(5 \mu \mathrm{M})$ in the presence and absence of GRS $(15 \mu \mathrm{M})$ were

578 measured in UV-capillaries using the Tycho NT6 (NanoTemper Technologies) where the protein

579 unfolding is followed by detecting the fluorescence of intrinsic tryptophan and tyrosine residues at

580 both emission wavelengths of $350 \mathrm{~nm}$ and $330 \mathrm{~nm}$.

\section{ACKNOWLEDGMENTS}

582 This work was supported by the US National Institute of Child Health and Human Development 583 (NICHD) U01HD085531 (to D.F.W. and R.W.). We acknowledge the funding support of the Research 584 Council of Norway (R\&D Project 261669 “Reversing antimicrobial resistance”) (to D.F.W.), the South

585 African Medical Research Council (to V.M. and D.F.W.); the National Research Foundation of South 586 Africa (to D.F.W. and V.M.); a Senior International Research Scholars grant from the Howard Hughes 587 Medical Institute (to V.M.); and a LUMC Fellowship (to M.H.L.). In addition, M.A.R. is grateful to the 588 South African National Research Foundation (NRF) for financial assistance during his PhD training 589 (grant no. 104683) as well as the Whitehead Scientific Travel Award. Z.A.M is grateful to the 590 University of Cape Town, the David and Elaine Potter Foundation Research Fellowship, and the Swiss 591 Government Excellence Research Scholarship for financial assistance during her PhD.

\section{SUPPLEMENTARY DATA}

593 Supplementary File 1 - Supplementary figures

594 Supplementary File 2 - Key reagents

595 Video 1 - Time-lapse microscopy of G-ImuB and mCherry-DnaN dual reporter. Representative

596 time-lapse movie of the reporter strain expressing G-ImuB and mCherry-DnaN. Bacteria were imaged 597 on fluorescence and phase channels for up to 36 hours at 10-minute intervals. Treatment with MMC $598(100 \mathrm{ng} / \mathrm{ml})$ was at $0-4.5$ hours. This experiment was repeated 6 times. Numbers indicate the hours 599 (h) elapsed in the time-lapse experiment. 7H9, Middlebrook 7H9/OADC. MMC, Mitomycin C. Scale 600 bar, $5 \mu \mathrm{m}$. G-ImuB, green; mCherry-DnaN, magenta; overlay, white. 
601 Video 2 - Time-lapse microscopy of V-ImuA' and mCherry-DnaN dual reporter. Representative

602 time-lapse movie of the reporter strain expressing V-ImuA' and mCherry-DnaN. Bacteria were

603 imaged on fluorescence and phase channels for up to 36 hours at 10-minute intervals. Treatment

604 with MMC $(100 \mathrm{ng} / \mathrm{ml})$ was at $0-4.5$ hours. This experiment was repeated 3 times. Numbers indicate

605 the hours (h) elapsed in the time-lapse experiment. 7H9, Middlebrook 7H9/OADC. MMC, Mitomycin

606 C. Scale bar, $5 \mu \mathrm{m}$. V-ImuA', green; mCherry-DnaN, magenta; overlay, white.

607 Video 3 - Time-lapse microscopy of G-DnaE2 and mCherry-DnaN dual reporter. Representative

608 time-lapse movie of the reporter strain expressing G-DnaE2 and mCherry-DnaN. Bacteria were

609 imaged on fluorescence and phase channels for up to 36 hours at 10-minute intervals. Treatment

610 with MMC $(100 \mathrm{ng} / \mathrm{ml})$ was at $0-4.5$ hours. This experiment was repeated 3 times. Numbers indicate

611 the hours (h) elapsed in the time-lapse experiment. 7H9, Middlebrook 7H9/OADC. MMC, Mitomycin

612 C. Scale bar, 5 m. G-DnaE2, green; mCherry-DnaN, magenta; overlay, white.

\section{REFERENCES}

614 Adefisayo, O.O., Dupuy, P., Bean, J.M. and Glickman, M.S. (2021). Division of labor between SOS and

615 PafBC in mycobacterial DNA repair and mutagenesis. bioRxiv, p. 2021.08.05.455301.

616 doi:10.1101/2021.08.05.455301.

617 Bargonetti, J., Champeil, E. and Tomasz, M. (2010). Differential toxicity of DNA adducts of mitomycin

618 C. Journal of Nucleic Acids, 698960. doi:10.4061/2010/698960.

619 Barrett, T.C., Mok, W.W.K.K., Murawski, A.M. and Brynildsen, M.P. (2019). Enhanced antibiotic resistance development from fluoroquinolone persisters after a single exposure to antibiotic. Nature Communications, 10(1), pp. 1-11. doi:10.1038/s41467-019-09058-4.

622 Boritsch, E.C. and Brosch, R. (2016). Evolution of Mycobacterium tuberculosis: New insights into pathogenicity and drug resistance. Microbiology Spectrum, (5), pp. 495-515. doi:10.1128/9781555819569.ch22.

625 Bosch, B., DeJesus, M.A., Poulton, N.C., Zhang, W., Engelhart, C.A., Zaveri, A., Lavalette, S., Ruecker, N., Trujillo, C., Wallach, J.B., Li, S., Ehrt, S., Chait, B.T., Schnappinger, D. and Rock, J.M. (2021).

Boshoff, H.I. and Mizrahi, V. (2000). Expression of Mycobacterium smegmatis pyrazinamidase in 
Boshoff, H.I.M.M., Reed, M.B., Barry, C.E. and Mizrahi, V. (2003). DnaE2 polymerase contributes to in vivo survival and the emergence of drug resistance in Mycobacterium tuberculosis. Cell, 113(2), pp. 183-193. doi:10.1016/S0092-8674(03)00270-8.

Brynildsrud, O.B., Pepperrell, C.S., Suffys, P., Grandjean, L., Monteserin, J., Debech, N., Bohlin, J., Alfsnes, K., Pettersson, J.O.H., Kirkeleite, I., Fandinho, F., da Silva, M.A., Perdigao, J., Portugal, I., Viveiros, M., Clark, T., Caws, M., Dunstan, S., Thai, P.V.K., Lopez, B., Ritacco, V., Kitchen, A., Brown, T.S., van Soolingen, D., O'Neill, M.B., Holt, K.E., Feil, E.J., Mathema, B., Balloux, F., Eldholm, V. (2018). Global expansion of Mycobacterium tuberculosis lineage 4 shaped by colonial migration and local adaptation. Science Advances, 4(10), pp. 1-12. doi:10.1126/sciadv.aat5869.

Bunting, K.A., Roe, S.M. and Pearl, L.H. (2003). Structural basis for recruitment of translesion DNA polymerase Pol IV/DinB to the $\beta$-clamp. EMBO Journal, 22(21), pp. 5883-5892. doi:10.1093/emboj/cdg568.

Chengalroyen, M.D., Mason, M.K., Borsellini, A., Tassoni, R., Abrahams, G.L., Lynch, S., Ahn, Y.-M.,

Burnouf, D.Y., Olieric, V., Wagner, J., Fujii, S., Reinbolt, J., Fuchs, R.P.P. and Dumas, P. (2004). Structural and biochemical analysis of sliding clamp/ligand interactions suggest a competition between replicative and translesion DNA polymerases. Journal of Molecular Biology, $335(5), \quad$ pp.

Cokol, M., Kuru, N., Bicak, E., Larkins-Ford, J. and Aldridge, B.B. (2017). Efficient measurement and factorization of high-order drug interactions in Mycobacterium tuberculosis. Science Advances, 3(10). doi:10.1126/sciadv.1701881.

Cormack, B.P., Valdivia, R.H. and Falkow, S. (1996). FACS-optimized mutants of the green fluorescent protein (GFP). Gene, 173(1), pp. 33-38. doi:10.1016/0378-1119(95)00685-0. 
Dhar, N. and Manina, G. (2015). Single-cell analysis of mycobacteria using microfluidics and timelapse microscopy. Methods in Molecular Biology, 1285, pp. 241-256.

Ducret, A., Quardokus, E. and Brun, Y. (2016). MicrobeJ, a tool for high throughput bacterial cell detection and quantitative analysis. Nature Microbiology, 1(7), pp. 1-14. doi:10.1038/nmicrobiol.2016.77.MicrobeJ.

van Eijk, E., Wittekoek, B., Kuijper, E.J. and Smits, W.K. (2017). DNA replication proteins as potential targets for antimicrobials in drug-resistant bacterial pathogens. Journal of Antimicrobial Chemotherapy, 72(5), pp. 1275-1284. doi:10.1093/JAC/DKW548.

Einhauer, A. and Jungbauer, A. (2001). The FLAGTM peptide, a versatile fusion tag for the purification of recombinant proteins. Journal of Biochemical and Biophysical Methods, 49(1-3), pp. 455465. doi:10.1016/S0165-022X(01)00213-5.

Erdem, A.L., Jaszczur, M., Bertram, J.G., Woodgate, R., Cox, M.M. and Goodman, M.F. (2014). DNA polymerase $\mathrm{V}$ activity is autoregulated by a novel intrinsic DNA-dependent ATPase. eLife, 2014(3). doi:10.7554/ELIFE.02384.

Erill, I., Campoy, S., Mazon, G. and Barbé, J. (2006). Dispersal and regulation of an adaptive mutagenesis cassette in the bacteria domain. Nucleic Acids Research, 34(1), pp. 66-77. doi:10.1093/nar/gkj412.

Farhat, M.R., Freschi, L., Calderon, R., Ioerger, T., Snyder, M., Meehan, C.J., de Jong, B., Rigouts, L., Sloutsky, A., Kaur, D., Sunyaev, S., van Soolingen, D., Shendure, J., Sacchettini, J. and Murray, M. (2019). GWAS for quantitative resistance phenotypes in Mycobacterium tuberculosis reveals resistance genes and regulatory regions. Nature Communications, 10(1). doi:10.1038/s41467019-10110-6.

Gagneux, S. (2018). Ecology and evolution of Mycobacterium tuberculosis. Nature Reviews Microbiology, 16(4), pp. 202-213. doi:10.1038/nrmicro.2018.8. doi:10.1038/nrg3664. levels of regulation imposed on Escherichia coli DNA polymerase V. DNA Repair, 44, pp. 42-50. doi:10.1016/J.DNAREP.2016.05.005.

Gray, T.A. and Derbyshire, K.M. (2018). Blending genomes: distributive conjugal transfer in 
mycobacteria, a sexier form of HGT. Molecular Microbiology, 108(6), pp. 601-613. doi:10.1111/mmi.13971.

Gruber, A.J., Erdem, A.L., Sabat, G., Karata, K., Jaszczur, M.M., Vo, D.D., Olsen, T.M., Woodgate, R., Goodman, M.F. and Cox, M.M. (2015). A RecA protein surface required for activation of DNA polymerase V. PLOS Genetics, 11(3), p. e1005066. doi:10.1371/JOURNAL.PGEN.1005066.

Hershberg, R., Lipatov, M., Small, P.M., Sheffer, H., Niemann, S., Homolka, S., Roach, J.C., Kremer, K., Petrov, D.A., Feldman, M.W. and Gagneux, S. (2008). High functional diversity in Mycobacterium tuberculosis driven by genetic drift and human demography. PLoS Biology, 6(12), pp. 2658-2671. doi:10.1371/journal.pbio.0060311.

Ippoliti, P.J., DeLateur, N.A., Jones, K.M. and Beuning, P.J. (2012). Multiple strategies for translesion synthesis in bacteria. Cells, 1(4), pp. 799-831. doi:10.3390/cells1040799.

Jaszczur, M.M., Vo, D.D., Stanciauskas, R., Bertram, J.G., Sikand, A., Cox, M.M., Woodgate, R., Mak, C.H., Pinaud, F. and Goodman, M.F. (2019). Conformational regulation of Escherichia coli DNA polymerase V by RecA and ATP. PLoS Genetics, 15(2), pp. 1-27. doi:10.1371/journal.pgen.1007956.

Joseph, A.M., Daw, S., Sadhir, I. and Badrinarayanan, A. (2021). Coordination between nucleotide excision repair and specialized polymerase Dnae2 action enables dna damage survival in nonreplicating bacteria. eLife, 10. doi:10.7554/ELIFE.67552.

Jiang, Q., Karata, K., Woodgate, R., Cox, M.M. and Goodman, M.F. (2009). The active form of DNA polymerase $\mathrm{V}$ is $\mathrm{UmuD}^{\prime} 2 \mathrm{C}-\mathrm{RecA}$-ATP. Nature, 460(7253), pp. 359-363. doi:10.1038/nature08178.

Kling, A., Lukat, P., Almeida, D.V., Bauer, A., Fontaine, E., Sordello, S., Zaburannyi, N., Herrmann, J., Wenzel, S., Konig, C., Ammerman, N.C., Barrio, M.B., Borchers, K., Bordon-Pallier, F., Bronstrup, M., Courtemanche, G., Gerlitz, M., Geslin, M., Hammann, P., Heinz, D.W., Hoffmann, H., Klieber, S., Kohlmann, M., Kurz, M., Lair, C., Matter, H., Nuermberger, E., Tyagi, S., Fraisse, L., Grosset, J., H., Lagrange, S., Müller, R.(2015). Targeting DnaN for tuberculosis therapy using novel griselimycins. Science, 348(6239), pp. 1106-1112. doi:10.1126/science.aaa4690.

Ley, S.., de Vos, M., Van Rie, A. and Warren, R.M. (2019.) Deciphering within-host microevolution of Mycobacterium tuberculosis through whole-genome sequencing: the phenotypic impact and way forward. Microbiology and Molecular Biology Reviews, 83(2), pp. 1-21.

730 Luna-Vargas, M.P.A., Christodoulou, E., Alfieri, A., van Dijk, W.J., Stadnik, M., Hibbert, R.G., Sahtoe, D.D., 
Clerici, M., Marco, V. De, Littler, D., Celie, P.H.N., Sixma, T.K. and Perrakis, A. (2011) Enabling high-throughput ligation-independent cloning and protein expression for the family of ubiquitin specific proteases. Journal of Structural Biology, 175(2), pp. 113-119. doi:10.1016/J.JSB.2011.03.017.

Maslowska, K.H., Makiela-Dzbenska, K. and Fijalkowska, I.J. (2019) The SOS system: A complex and tightly regulated response to DNA damage. Environmental and Molecular Mutagenesis, 60(4), pp. 368-384. doi:10.1002/em.22267.

McHenry, C.S. (2011). Breaking the rules: bacteria that use several DNA polymerase IIIs. EMBO reports, 12(5), pp. 408-414. doi:10.1038/embor.2011.51.

Merrikh, H. and Kohli, R.M. (2020). Targeting evolution to inhibit antibiotic resistance. FEBS Journal, 287(20), pp. 4341-4353. doi:10.1111/febs.15370.

Minias, A., Brzostek, A. and Dziadek, J.J. (2018). Targeting DNA repair systems in antitubercular drug development. Current Medicinal doi:10.2174/0929867325666180129093546.

Mittal, P., Sinha, R., Kumar, A., Singh, P., Ngasainao, M.R., Singh, A. and Singh, I.K. (2020). Focusing on DNA repair and damage tolerance mechanisms in Mycobacterium tuberculosis: An emerging therapeutic theme. Current Topics in Medicinal Chemistry, 20(5), pp. 390-408. doi:10.2174/1568026620666200110114322.

Nagai, T., Ibata, K., Park, E.S., Kubota, M., Mikoshiba, K. and Miyawaki, A. (2002). A variant of yellow fluorescent protein with fast and efficient maturation for cell-biological applications. Nature Biotechnology, 20(1), pp. 87-90. doi:10.1038/nbt0102-87.

Paez Segala, M.G., Sun, M., Shtengel, G., Viswanathan, S., Baird, M., Macklin, J., Patel, R., Allen, J., Howe,

Payne, J.L., Menardo, F., Trauner, A., Borrell, S., Gygli, S.M., Loiseau, C., Gagneux, S. and Hall, A.R. (2019). Transition bias influences the evolution of antibiotic resistance in Mycobacterium tuberculosis. PLoS, 17(5), pp. 1-23. doi:10.1101/421651. Miller, S.I., Sherman, D.R. and Merrikh, H. (2019). Inhibiting the evolution of antibiotic resistance. Molecular Cell, 73(1), pp. 157-165.e5. doi:10.1016/j.molcel.2018.10.015.

Reiche, M.A., Warner, D.F. and Mizrahi, V. (2017). Targeting DNA replication and repair for the development of novel therapeutics against tuberculosis. Frontiers in Molecular Biosciences, 
4(November), pp. 1-18. doi:10.3389/fmolb.2017.00075.

Renzette, N., Gumlaw, N., Nordman, J.T., Krieger, M., Yeh, S.P., Long, E., Centore, R., Boonsombat, R. and Sandler, S.J. (2005). Localization of RecA in Escherichia coli K-12 using RecA-GFP. Molecular Microbiology, 57(4), pp. 1074-1085. doi:10.1111/j.1365-2958.2005.04755.x.

Revitt-Mills, S.A. and Robinson, A. (2020). Antibiotic-induced mutagenesis: Under the microscope. Frontiers in Microbiology, 0, p. 2611. doi:10.3389/FMICB.2020.585175.

Riojas, M.A., McGough, K.J., Rider-Riojas, C.J., Rastogi, N. and Hazbón, M.H. (2018). Phylogenomic analysis of the species of the Mycobacterium tuberculosis complex demonstrates that Mycobacterium africanum, Mycobacterium bovis, Mycobacterium caprae, Mycobacterium microti and Mycobacterium pinnipedii are later heterotypic synonyms of Mycobacterium tuberculosis. International Journal of Systematic and Evolutionary Microbiology, 68(1), pp. 324332. doi:10.1099/IJSEM.0.002507.

Robinson, A., McDonald, J.P., Caldas, V.E.A., Patel, M., Wood, E.A., Punter, C.M., Ghodke, H., Cox, M.M.,

Santi, I., Dhar, N., Bousbaine, D., Wakamoto, Y. and McKinney, J.D. (2013). Single-cell dynamics of the chromosome replication and cell division cycles in mycobacteria. Nature Communications, 4(May), pp. 1-10. doi:10.1038/ncomms3470.

Santi, I. and McKinney, J.D. (2015). Chromosome organization and replisome dynamics in Mycobacterium smegmatis. mBio, 6(1). doi:10.1128/mBio.01999-14.

Schindelin, J., Arganda-Carreras, I., Frise, E., Kaynig, V., Longair, M., Pietzsch, T., Preibisch, S., Rueden, C., Saalfeld, S., Schmid, B., Tinevez, J.-Y., White, D.J., Hartenstein, V., Eliceiri, K., Tomancak, P. and Cardona, A. (2012). Fiji: an open-source platform for biological-image analysis. Nature Methods, 9(7), pp. 676-682. doi:10.1038/nmeth.2019.

Shtengel, G., Galbraith, J.A., Galbraith, C.G., Lippincott-Schwartz, J., Gillette, J.M., Manley, S., Sougrat, R., Waterman, C.M., Kanchanawong, P., Davidson, M.W., Fetter, R.D. and Hess, H.F. (2009). Interferometric fluorescent super-resolution microscopy resolves 3D cellular ultrastructure. Proceedings of the National Academy of Sciences of the United States of America, 106(9), pp. 3125-3130. doi:10.1073/pnas.0813131106.

Singh, A. (2017). Guardians of the mycobacterial genome: A review on DNA repair systems in Mycobacterium tuberculosis. Microbiology, 163(12), pp. 1740-1758. doi:10.1099/mic.0.000578.

796 Smith, P.A. and Romesberg, F.E. (2007). Combating bacteria and drug resistance by inhibiting 
mechanisms of persistence and adaptation. Nature Chemical Biology, 3(9), pp. 549-556. doi:10.1038/nchembio.2007.27.

Timinskas, K., Balvočiute, M., Timinskas, A. and Venclovas, Č. (2014). Comprehensive analysis of DNA polymerase III $\alpha$ subunits and their homologs in bacterial genomes. Nucleic Acids Research, 42(3), pp. 1393-1413. doi:10.1093/nar/gkt900.

Timinskas, K. and Venclovas, Č. (2019). New insights into the structures and interactions of bacterial Y-family DNA polymerases. Nucleic Acids Research, 47(9), pp. 4383-4405. doi:10.1093/nar/gkz198.

Tomasz, M. (1995). Mitomycin C: small, fast and deadly (but very selective). Chemistry and Biology, pp. 575-579. doi:10.1016/1074-5521(95)90120-5.

Veening, J.-W. and Blokesch, M. (2017). Interbacterial predation as a strategy for DNA acquisition in naturally competent bacteria. Nature Reviews Microbiology, 15(10), pp. 621-629. doi:10.1038/nrmicro.2017.66.

Wakamoto, Y., Dhar, N., Chait, R., Schneider, K., Signorino-Gelo, F., Leibler, S. and McKinney, J.D. (2013). Dynamic persistence of antibiotic-stressed mycobacteria. Science, 339, pp. 91-95.

Warner, D.F., Koch, A. and Mizrahi, V. (2015). Diversity and disease pathogenesis in Mycobacterium tuberculosis. Trends in Microbiology, pp. 14-21. doi:10.1016/j.tim.2014.10.005.

Warner, D.F., Ndwandwe, D.E., Abrahams, G.L., Kana, B.D., Machowski, E.E., Venclovas, Č. and Mizrahi, V. (2010). Essential roles for imuA'- and $i m u B$-encoded accessory factors in DnaE2-dependent mutagenesis in Mycobacterium tuberculosis. Proceedings of the National Academy of Sciences of the United States of America, 107(29), pp. 13093-13098. doi:10.1073/pnas.1002614107.

824 von Wintersdorff, C.J.H., Penders, J., van Niekerk, J.M., Mills, N.D., Majumder, S., van Alphen, L.B., Savelkoul, P.H.M. and Wolffs, P.F.G. (2016). Dissemination of antimicrobial resistance in microbial ecosystems through horizontal gene transfer. Frontiers in Microbiology, 0(FEB), p. 